\title{
A physiological approach to the timing of umbilical cord clamping at birth
}

\author{
Stuart B Hooper, ${ }^{1,2}$ Graeme R Polglase, ${ }^{1,2}$ Arjan B te Pas ${ }^{3}$
}

${ }^{1}$ Ritchie Centre, MIMR-PHI Institute of Medical Research, Monash University, Melbourne, Victoria, Australia

${ }^{2}$ Department of Obstetrics \&

Gynaecology, Monash

University, Melbourne, Victoria, Australia

${ }^{3}$ Department of Pediatrics, Leiden University Medical Centre, Leiden, the

Netherlands

\section{Correspondence to}

Professor Stuart B Hooper, The Ritchie Centre, MIMR-PHI Institute of Medical Research, 27-31 Wright St, Clayton, VIC 3168, Australia; stuart.hooper@monash.edu

Received 3 October 2014 Revised 27 November 2014 Accepted 1 December 2014 Published Online First 24 December 2014

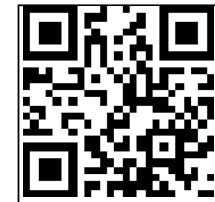

Editor's choice Scan to access mor free content

\section{CrossMark}

To cite: Hooper $S B$, Polglase GR, te Pas AB. Arch Dis Child Fetal Neonatal Ed 2015;100: F355-F360.

\section{ABSTRACT}

Umbilical cord clamping at birth has a major impact on an infant's cardiovascular system that varies in significance depending upon whether the infant has commenced breathing. As umbilical venous return is a major source of preload for the left ventricle during fetal life, recent experimental evidence has shown that clamping the umbilical cord severely limits cardiac venous return in the absence of pulmonary ventilation. As a result, cardiac output greatly reduces and remains low until breathing commences. Once the infant begins breathing, aeration of the lung triggers a large increase in pulmonary blood flow, which replaces umbilical venous return as the source of preload for the left ventricle. As a result, cardiac output markedly increases, as indicated by an increase in heart rate immediately after birth. Thus, infants born apnoeic and hypoxic and have their cords immediately clamped, are likely to have a restricted cardiac output combined with hypoxia. As increased cardiac output is a major physiological defence mechanism that counteracts the effects of hypoxaemia, limiting the increase in cardiac output exposes the infant to ischaemia along with hypoxia. However, if the infant commences breathing, aerates its lungs and increases pulmonary blood flow before the umbilical cord is clamped, then pulmonary venous return can immediately take over the supply of left ventricular preload upon cord clamping. As a result, there is no intervening period of reduced preload and cardiac output and the large swings in arterial pressures and flows are reduced leading to a more stable circulatory transition.

\section{INTRODUCTION}

Umbilical cord clamping (UCC) at birth is much more than a symbolic separation of the infant from the mother. Potentially, UCC can cause a major disturbance to the infant's cardiovascular system, placing the newborn at increased risk of death or brain injury. ${ }^{1}$ To survive birth, the infant must immediately initiate pulmonary gas exchange and redirect output from the right ventricle through the lungs. To achieve this, the liquid-filled lungs must first aerate so that air can penetrate into the distal airways and gas exchange can commence. This event triggers a large increase in pulmonary blood flow (PBF) that increases pulmonary venous return allowing the pulmonary circulation to take over from the umbilical circulation as the source of preload for the left ventricle. As the source of preload for the left ventricle is predominantly derived from the umbilical circulation during fetal life, ${ }^{2}$ UCC at birth can severely restrict the supply of preload to the left ventricle, resulting in a 50\% reduction in ventricular output. ${ }^{3}{ }^{3}$ The loss of preload and reduction in cardiac output represent significant challenges for the infant, particularly as it coincides with the switch to pulmonary gas exchange. As it can take many minutes for infants to aerate their lungs and increase PBF, these newborns are at a high risk of a combined hypoxic/ ischaemic event during this time. Recent experimental evidence suggests that delaying UCC until after the infant has commenced breathing, may greatly reduce this risk. ${ }^{1}$

Until recently, the benefits and risks associated with delayed UCC have mainly been attributed to net placento-fetal blood transfusion, resulting in an increase in neonatal blood volume. The placenta to fetus blood transfer is thought to be time dependent, beginning within $15 \mathrm{~s}$ and ceasing at $\sim 3 \mathrm{~min}$ after delivery, and to occur in a stepwise pattern, possibly due to uterine contractions. ${ }^{4}$ As a result, there has been much debate about the potential clinical risks and benefits of placental to infant blood transfusion during delayed UCC, which has been the subject of many recent reviews. ${ }^{5} 6$ However, net placental to infant blood transfusion does not readily explain some of the observations commonly associated with delayed UCC. As recent experimental evidence suggests that aerating the lungs and increasing PBF before UCC greatly stabilises the cardiovascular transition at birth, there is now a need to reconsider the timing of UCC with respect to the physiological transition at birth. ${ }^{7}$ As this is a subject that has received relatively little attention, this review will focus on the science underpinning the effect of UCC on the physiological changes at birth.

\section{FETAL CARDIOVASCULAR FUNCTION BEFORE BIRTH}

The functional structure of the fetal cardiovascular system is fundamentally different to that of the adult, but rapidly transforms into the adult phenotype within minutes of birth. ${ }^{2} 8$ In contrast to adults, the majority of right ventricular output in the fetus bypasses the lungs and flows from the main pulmonary artery and into the descending aorta via the ductus arteriosus (DA; figure 1). This is largely due to the high pulmonary vascular resistance (PVR) before birth, which results in a very low $\mathrm{PBF}^{2}{ }^{8}$ Animal studies have shown that flow into the left and right pulmonary arteries only occurs briefly during early to mid systole. ${ }^{3}$ During late systole and throughout diastole, PBF is retrograde, whereby blood flows away from the lungs and exits the pulmonary circulation by passing through the DA and entering the systemic circulation (figures 1 and 2). ${ }^{3}$ This retrograde flow of blood in the pulmonary arteries accounts for the high blood flow through the DA during diastole 
Fetus (A)

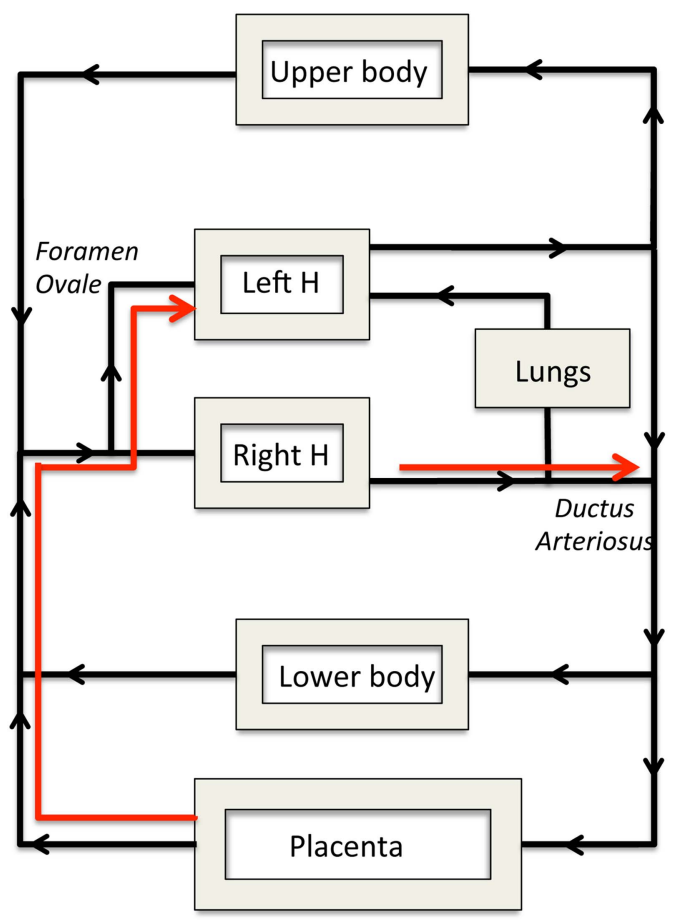

Newborn (B)

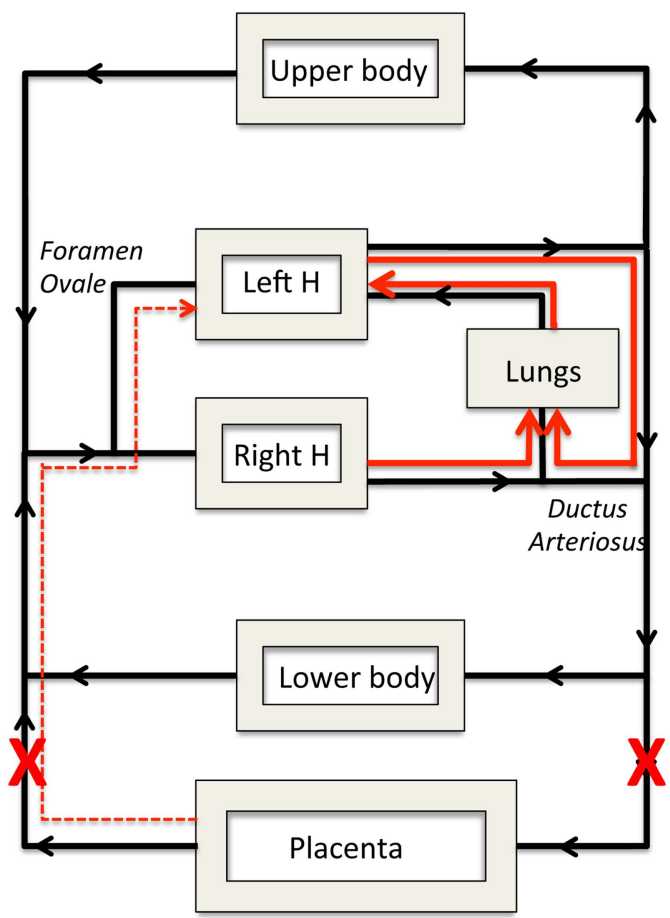

Figure 1 A schematic of the fetal circulation before birth and the changes in flow that occur after birth. Before birth the major supply of preload for the left ventricle is derived from the placental circulation, which passes from the umbilical vein through the ductus venosus, inferior vena cava and foramen ovale to directly enter the left side of the heart; thereby bypassing the right side of the heart and the lungs ( $A$; pathway shown by red arrow). As most blood exiting the right ventricle passes through the ductus arteriosus ( $A$; red arrow) and enters the descending aorta, very little blood flows into the fetal lungs before birth. After birth, the supply of blood for the left ventricle derived from the placental circulation is lost ( $B$; broken red arrow) and so preload for the left ventricle becomes dependent on pulmonary venous return ( $B$; red arrow). For this to occur, the lung must first aerate, which triggers a decrease in pulmonary vascular resistance, allowing all of right ventricular output to pass through the lungs (B; red arrow). In addition, flow through the ductus arteriosus reverses so that left ventricular output becomes a major contributor to pulmonary blood flow and, therefore to pulmonary venous return as well.

when flow in the main pulmonary trunk is zero. ${ }^{2} 3$ As a result, blood flows continuously through the DA, flowing from the pulmonary circulation into the systemic circulation (termed right-to-left (R-L) shunting), throughout the cardiac cycle. Evidence for similar flow patterns have been observed in human fetuses using ultrasonography ${ }^{10}$ or more recently by MRI. ${ }^{11}$

A consequence of the very low PBF during fetal life is that pulmonary venous return is also low and so left ventricular preload depends primarily on umbilical venous supply. That is, umbilical venous blood either passes through the ductus venosus (DV) or the liver before entering the inferior vena cava (figure 1) and returning to the heart. ${ }^{12}$ Animal studies have shown that a high proportion of this venous return, particularly the oxygenated umbilical vein derived blood that passes through the DV, bypasses the right ventricle and lungs by traversing through the foramen ovale (FO) to directly enter the left atrium. ${ }^{12}$ As a result, umbilical venous blood is a major source of preload for the left ventricle, with much of it passing through the DV and FO. This explains why left ventricular output, which supplies the preductal arteries, has higher oxygen levels than postductal arteries. ${ }^{12}$

\section{LUNG AERATION AND THE INCREASE IN PBF AT BIRTH}

One of the first and greatest challenges that an infant faces at birth is lung aeration and the onset of pulmonary gas exchange, which triggers many of the other adaptive changes required for postnatal survival. Before birth the airways are filled with liquid that is secreted by the lungs and exits the lungs by flowing out of the trachea. ${ }^{13}$ While this liquid provides a vital role for fetal lung growth and development, ${ }^{13}$ at birth it acts as an obstacle that restricts the entry of air and, thereby, the onset of pulmonary gas exchange. As such, at birth the airways must be cleared of liquid to enable air to penetrate down into the gas exchange regions of the lung so that gas exchange can commence. While a number of mechanisms contribute to airway liquid clearance at birth, the primary mechanisms involved will likely depend on the timing and mode of delivery. ${ }^{14}{ }^{15}$ Nevertheless, recent imaging studies in newborn rabbits have demonstrated that after birth, any liquid remaining in the airways is predominantly cleared by pressure gradients generated during inspiration, ${ }^{16-18}$ which drives liquid movement from the airways into alveolar tissue, where it is cleared by the lymphatics and blood vessels. ${ }^{19}$

The vital role of lung aeration in the fetal to neonatal transition is underpinned by the onset of pulmonary gas exchange and by the fact that it triggers a huge decrease in PVR and an increase in PBF. ${ }^{3}{ }^{20}$ This decrease in PVR and increase in PBF, along with UCC, are responsible for the transition of the fetal circulation into the adult phenotype. While this relationship between lung aeration and the increase in PBF at birth is well established, until recently it was assumed that it was primarily mediated by an increase in oxygenation. ${ }^{8}$ Indeed it is well known that increases and decreases in oxygenation increase and decrease PBF, respectively. ${ }^{21}$ However, previous experimental studies have questioned whether increased oxygenation has an overaching dominant role or is simply a contributor that 
Before birth

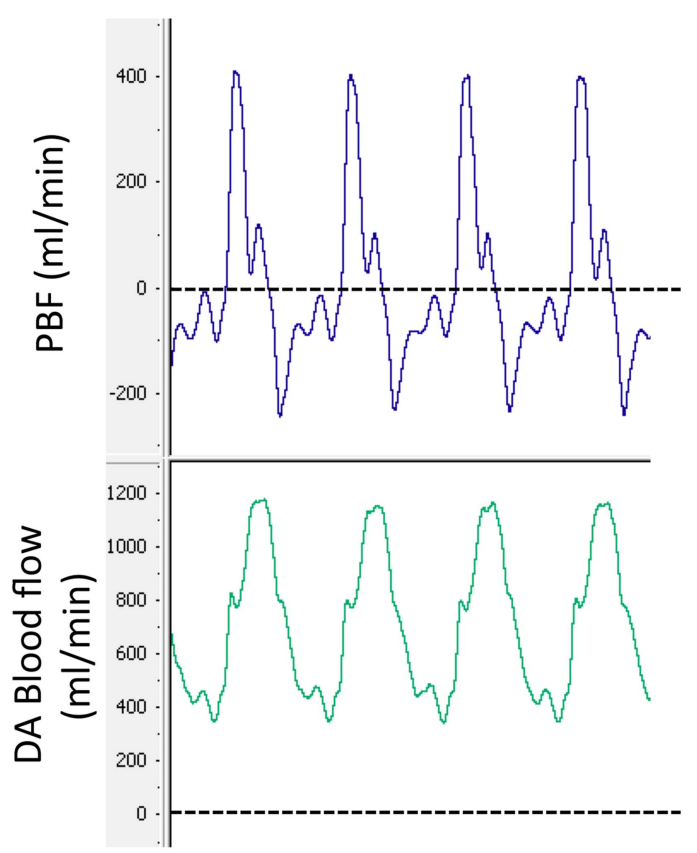

After birth (<10 mins)

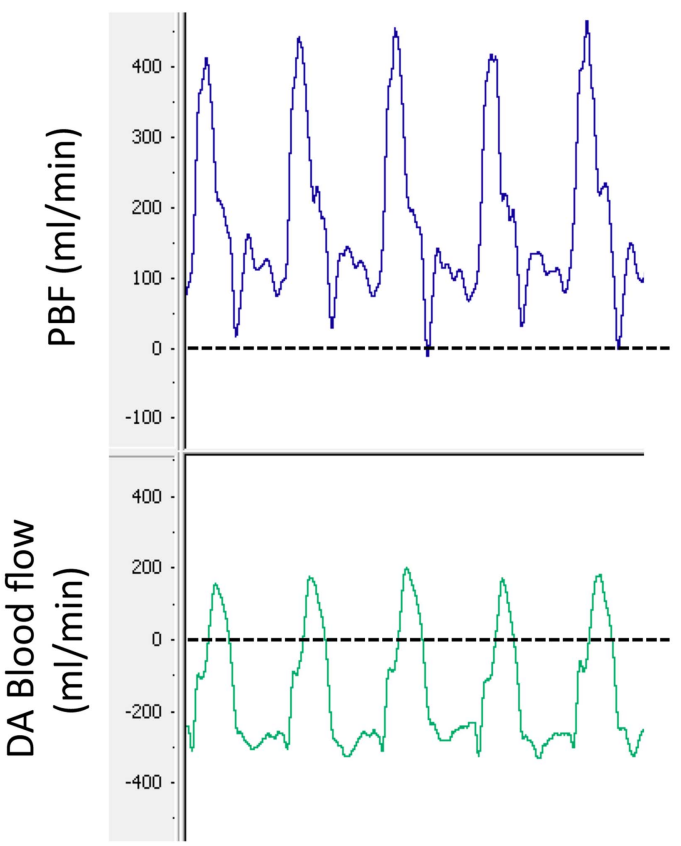

Figure 2 Blood flow waveforms in the left pulmonary artery and in the ductus arteriosus (DA) before and immediately after birth. Before birth, pulmonary blood flow (PBF) flows towards the lungs (positive flow) only briefly during systole and then during late systole and throughout most of diastole, PBF is mostly retrograde (negative value), flowing away from the lungs and passing through the DA. This retrograde PBF accounts for the high levels of diastolic flow in the DA. After birth, the decrease in pulmonary vascular resistance facilitates antegrade flow in the pulmonary arteries throughout the cardiac cycle, with relatively high flows occurring even during diastole. These diastolic flows are due to left-to-right shunting (indicated by negative flows) through the DA, contributing to flow during this time. Although net blood flow across the DA is predominantly left-to-right, the flow waveform demonstrates distinct bidirectional characteristics due to the changing pressure gradient across the DA associated with the cardiac cycle.

provides the fine control for ventilation/perfusion matching after birth. ${ }^{22}$ For instance, ventilation of lambs with a hypoxic gas mixture has been shown to increase PBF without an increase in oxygenation. ${ }^{23}$ Similarly, ventilation of lambs after birth with strategies that either increase or reduce arterial oxygenation levels (compared with the fetal state) produced similar increases in PBF, indicating that the PBF increase was independent of oxygenation status. ${ }^{24}$ More recently, an imaging study in newborn rabbits has used phase contrast X-ray imaging combined with angiography to show that unilateral aeration of the lung causes a global increase in $\mathrm{PBF}^{22}$ Although this leads to a large ventilation perfusion mismatch in unventilated lung regions, delinking the spatial relationship between lung aeration and the increase in PBF at birth has some adaptive advantages (see below). This study ${ }^{22}$ has potentially uncovered a previously unknown mechanism, which may be the primary driver for the increase in PBF induced by lung aeration.

\section{CARDIOVASCULAR CONSEQUENCES OF UCC AND LUNG AERATION AT BIRTH}

In normal uncomplicated pregnancies before birth, the placental circulation is a low resistance, highly compliant vascular bed that receives a large proportion $(30-50 \%)$ of total cardiac output. ${ }^{12}$ As a result, UCC at birth in lambs causes an immediate (stepwise) increase in systemic peripheral resistance that results in a rapid (within four heartbeats or $\sim 1 \mathrm{~s}$ ) increase $(\sim 30 \%)$ in arterial pressure (figure 3$).{ }^{1}$ Because the increase in arterial pressure is so rapid, it also causes a similar, pressure driven, increase in cerebral blood flow. ${ }^{1}$ This indicates that within this time frame, the cerebral circulation is pressure passive and vulnerable to large changes in flow in response to rapid fluctuations in pressure. Indeed, as UCC also reduces cardiac output (see below) this elevation in systemic arterial pressure and cerebral blood flow is transient and followed by a rapid reduction and then a rapid increase again after ventilation onset (figure 3 ). ${ }^{1}$

Depending upon the gestational age of the fetus and its relative size compared with the placenta, between $30-50 \%$ of total fetal combined ventricular output flows through the umbilical circulation. ${ }^{12}$ As such, UCC causes a profound reduction in venous return and ventricular preload. Indeed, immediate UCC at birth in preterm lambs causes an immediate $50 \%$ reduction in right ventricular output (figure 3). ${ }^{13}$ Cardiac output remains low until the lung aerates and PBF increases venous return and preload for the left ventricle and possibly the right ventricle via the FO (figure 1). While it is thought that flow through the FO is unidirectional, mostly R-L (from right to left atrium), ${ }^{25}$ the rapid increase in right ventricular output associated with lung aeration (figure 3) suggests that some L-R (left to right atrium) flow may occur at birth in lambs. ${ }^{1}$ This is supported by Doppler ultrasonography in lambs (unpublished observations) and the finding that significant L-R flow through the FO can occur in humans. $^{26}$

The decrease in PVR associated with lung aeration after birth has a number of consequences, most notably an increase in PBF. This increase in PBF results from a redirection of right ventricular output to pass entirely through the lungs and a reversal in net flow through the DA (figure 1). Both of these events occur because downstream resistance in the pulmonary circulation decreases below the resistance in the lower body. As a result, 


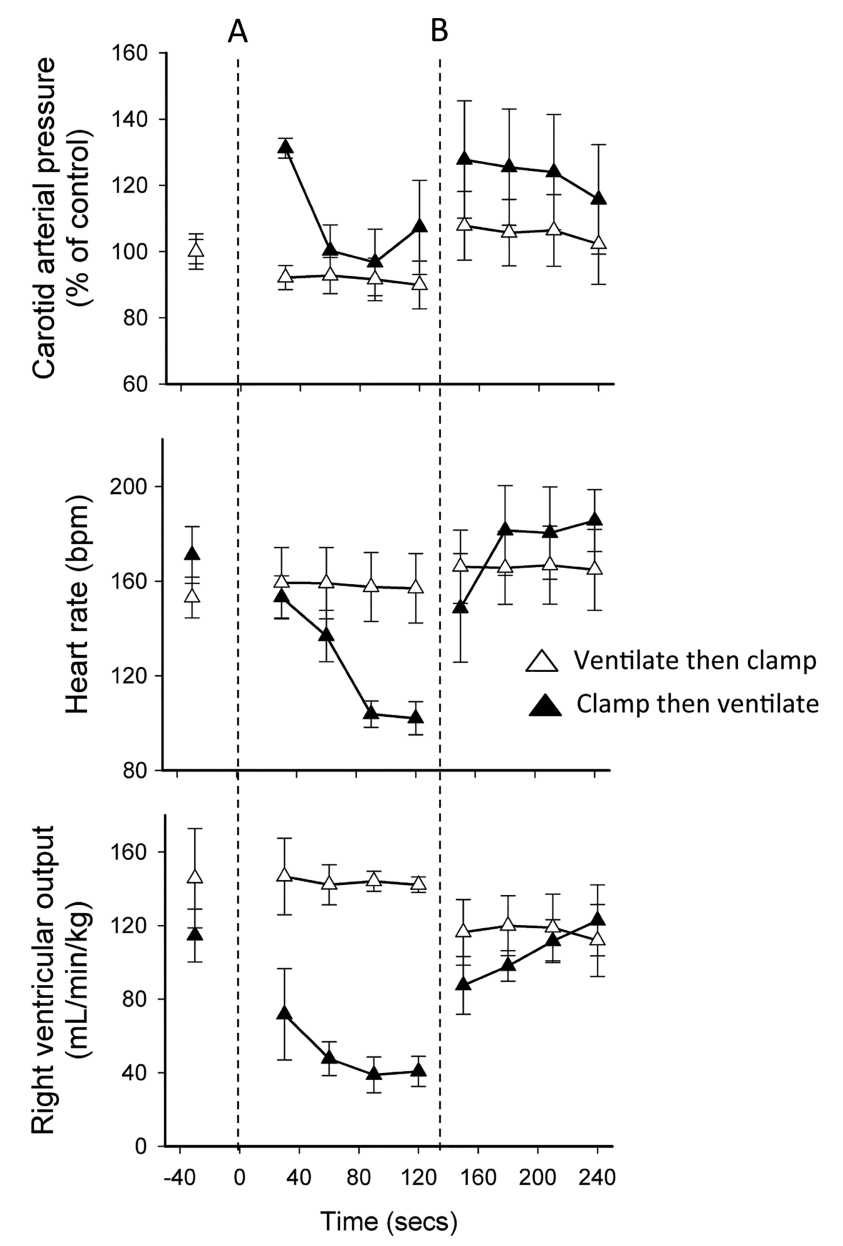

Figure 3 Carotid arterial pressure, heart rate and right ventricular output measured in newborn lambs that either had their umbilical cords clamped 1-2 min before ventilation was commenced (clamp first; closed triangles) or were ventilated and pulmonary blood flow allowed to increase before their cords were clamped (vent first; open triangles). The broken line $(A)$ indicates either when cord clamping occurred in the clamp first group or ventilation commenced in the vent first group. The broken line (B) indicates when either clamping occurred in the vent first group or when ventilation commenced in the clamp first group. Data were obtained from Bhatt et al ${ }^{1}$ and redrawn.

blood preferentially flows from L-R through the DA, from the aorta and into the pulmonary circulation, down the path of least resistance (figure 1). While net flow is from L-R, instantaneous flow is largely bidirectional (figure 2), which is likely due to differences in timing for when the pressure waves, emanating from the left and right ventricles, reach either end of the DA. ${ }^{3} 2728$ That is, the pressure wave leaving the right ventricle reaches the pulmonary artery/DA junction before the pressure wave from the left ventricle reaches the DA/aorta junction. This creates a pulmonary artery to aorta pressure gradient that facilitates R-L flow early in systole (figure 2). However, when the pressure wave from the left ventricle reaches the DA/aorta junction, the pressure gradient across the DA reverses causing L-R flow, which predominates in late systole and throughout diastole. The L-R flow through the DA is responsible for up to $50 \%$ of the increase in PBF at birth and is entirely responsible for the high rates of PBF that have been observed during diastole immediately after birth (figure 2); flow in the main pulmonary trunk reaches zero at this time.

The increase in PBF immediately after birth plays a critical role in the transition to newborn life by facilitating pulmonary gas exchange and by supplying venous return (preload) for the left ventricle. ${ }^{14}$ As indicated above, before birth, the source of preload is primarily supplied by umbilical venous return and so UCC at birth causes a dramatic reduction in ventricular preload and a decrease in cardiac output (figure 3 ). ${ }^{13}$ In lambs, this reduction persists until the lung aerates and PBF increases to take over the role of supplying preload to the left ventricle (figure 3). ${ }^{1}$ Thus, if there is a significant delay between UCC and lung aeration after birth, the infant will be exposed to a hypoxic episode, and to a protracted period of low cardiac output. The primary physiological mechanism that is available to protect vital organs such as the brain from hypoxic episodes involves an increase and redistribution in cardiac output, which results in a large increase in cerebral blood flow. ${ }^{12} 2930$ As such, the period between cord clamping and ventilation onset, when cardiac output is compromised, represents a period when the infant is at significant risk of hypoxic/ischaemic brain injury (figure 3). On the other hand, infants that are not exposed to a hypoxic episode following immediate cord clamping will be exposed to large swings in arterial pressure and cerebral blood flow (figure 3), which increases the risk of perinatal brain injury. ${ }^{31}$ This is because cord clamping rapidly increases arterial pressure and cerebral blood flow and this is followed by a reduction in pressure and flow due to a reduction in cardiac output. Then upon lung aeration, PBF increases which restores ventricular preload and cardiac output, resulting in a rebound increase in arterial pressure and cerebral blood flow. ${ }^{1}$ This can be avoided if UCC occurs after the lungs have aerated and ventilation has commenced (figure 3).

\section{CARDIOVASCULAR BENEFITS OF LUNG AERATION BEFORE UCC}

In view of the infant's need to switch venous return and ventricular preload from umbilical to pulmonary venous return, it is logical to initiate pulmonary ventilation and increase PBF before UCC. The increase in PBF after birth usually begins quickly (within minutes), depending upon the rate at which lung aeration commences, and in ventilated lambs it can take 5-10 min before the PBF reaches a maximum. ${ }^{3}$ During this period of increasing PBF, the increase in pulmonary venous return and left ventricular preload simultaneously restores cardiac output, ${ }^{13}$ or at least the capacity to increase cardiac output if required. As such, it is logical that the umbilical circulation remains intact during this time so that the reduction in venous return and cardiac output associated with the loss of umbilical venous flow is minimised (figure 1). When PBF increases before UCC, the supply of ventricular preload can immediately switch from umbilical to pulmonary venous return without any diminution to supply. ${ }^{1}$ As a result, there is no loss in cardiac output and the large swings in arterial pressure and cerebral blood flow associated with UCC are greatly mitigated (figure 3). Furthermore, the rapid increase in arterial pressure (over four heartbeats) that occurs in response to UCC is also greatly reduced. ${ }^{1}$ This is because the low resistance pulmonary circulation, via L-R flow through the DA, can immediately become an alternate pathway for left ventricular output.

In theory, and under controlled experimental settings, it is evident that there is considerable potential benefit for the infant if the lungs aerate and PBF increases before UCC. However, in reality there are several practical issues that are likely to impact on the physiological benefits for the infant. However, none of these relate to the practicalities of resuscitating an infant next to the mother following either vaginal or caesarean section delivery. Instead, these relate to the vertical positioning of the infant, 
relative to the mother, and the timing of uterotonic administration, as this could greatly impact upon cardiovascular benefits of initiating lung aeration before UCC. Furthermore, in severely asphyxic bradycardic infants requiring chest compressions, it is unclear whether delayed cord clamping would be beneficial or detrimental to the resuscitation. Indeed, the presence of a persisting low resistance placental circulation may reduce or restrict the increase in diastolic pressure required to restore spontaneous circulation.

Much attention has focused on the obstetric management of the third stage of labour, with much of this focus centred on maternal welfare and reducing the risk of postpartum haemorrhage. Until recently, it was widely recommended that the mother be given a uterotonic (eg, syntocinon) upon delivery of the infant's anterior shoulder, which is followed by immediate UCC and gentle cord traction to hasten delivery of the placenta. $^{32} 33$ While this management strategy reduces the risk of postpartum haemorrhage, mainly due to uterotonic administration, ${ }^{34}$ it is also associated with a significant reduction in infant birth weight, which is thought to be due to a reduction in infant blood volume. ${ }^{32}$ However, as oxytocin is equally effective when given following delivery of the placenta, ${ }^{33}$ it may be preferential to administer it at the end of the third stage to avoid interfering with the placental circulation during delayed cord clamping.

Clinical studies suggest that, while the umbilical circulation remains intact, uterine contractions 'squeeze' umbilical blood towards the infant and enhance the blood transfusion associated with delayed UCC. ${ }^{35}$ However, this suggestion is not consistent with the interpretation of fetal heart rate variability associated with uterine contractions during the second stage of labour. ${ }^{36}$ Differential occlusion of umbilical venous and arterial vessels (intraplacental) during an uterine contraction is thought to cause the placental accumulation of blood. This is due to the earlier occlusion and later release of the more compliant venous vessels, compared with the arteries, leading to increased venous return and an increase in heart rate at the end of the contraction. ${ }^{36}$ As a sustained and intense uterine contraction causes umbilical blood flow to reduce or cease, oxytocin administration during delayed UCC will likely negate the benefits of this procedure.

The effect of positioning the infant above or below the placenta is another factor that could influence the cardiovascular transition during delayed UCC. It is assumed that placing the infant above the placenta increases blood flow into the placenta whereas placing the infant below the placenta increases blood flow into the infant. This assumption is supported by studies ${ }^{4} 37$ showing that placental transfusion is maximised when neonates are placed below the placenta. ${ }^{37}$ While the effects were explained by the ability of gravity to assist or reduce the placental to infant blood transfusion, the haemodynamics are likely to be considerably more complex. Nevertheless, a recent non-inferiority clinical trial has found no adverse effects (on infant weight) of placing the infant above the placenta, ${ }^{39}$ which is consistent with the current common practice of placing the infant on the mother's abdomen or chest after birth. While this does not appear to influence infant outcome,${ }^{39}$ the science underpinning this practice has not been investigated.

\section{SUMMARY}

While UCC is widely considered to be an innocuous act, it is now apparent that this act is only innocuous if it is done at the right time. Indeed, the appropriate time for UCC is after the infant has aerated its lungs, commenced breathing and PBF has increased to sustain ventricular preload and cardiac output.
Otherwise, if there is a long delay between UCC and lung aeration, the infant will be exposed to a hypoxic episode superimposed on top of a period of severely restricted cardiac function. The combined effects of these two adverse events are potentially catastrophic, leading to a severe hypoxic/ischaemic event. Although the timing of UCC at birth has been an issue of debate for centuries, recently the debate on this topic has almost entirely focused on placental to infant blood transfusion. While higher haemoglobin levels may have important implications for long-term infant outcomes, this issue is not the only, or perhaps even the most important issue, that concerns the appropriate timing of UCC at birth. We suggest that the focus of the debate should shift away from a time-based approach to a physiologically based approach for the timing of UCC. Indeed, due to the huge variability between individual infants there appears to be little logic in deciding to clamp the cord based on an arbitrarily set period of time. Rather, if the decision is based on the infant's physiology, the timing can be tailored to suit the infant's needs.

Contributors All authors have contributed significantly to the development and writing of this manuscript.

Funding This research was supported by a NH\&MRC Program Grant (606789) and Research Fellowships (GRP: 1026890 and SBH: 545921), a Rebecca L. Cooper Medical Research Foundation Fellowship (GRP), a Eunice Kennedy Shriver National Institute Of Child Health \& Human Development of the National Institutes of Health (Award Number R01HD072848), The Financial Markets Foundation for Children and the Victorian Government's Operational Infrastructure Support Program.

Competing interests None.

Provenance and peer review Commissioned; externally peer reviewed.

\section{REFERENCES}

1 Bhatt $\mathrm{S}$, Alison B, Wallace EM, et al. Delaying cord clamping until ventilation onset improves cardiovascular function at birth in preterm lambs. J Physiol 2013;591:2113-26.

2 Rudolph AM. Fetal and neonatal pulmonary circulation. Annu Rev Physiol 1979;41:383-95.

3 Crossley KJ, Allison BJ, Polglase GR, et al. Dynamic changes in the direction of blood flow through the ductus arteriosus at birth. J Physiol 2009;587(Pt 19):4695-704.

4 Yao AC, Moinian M, Lind J. Distribution of blood between infant and placenta after birth. Lancet 1969;2:871-3.

5 Niermeyer S, Velaphi S. Promoting physiologic transition at birth: Re-examining resuscitation and the timing of cord clamping. Semin Fetal Neonatal Med 2013:18:385-92.

6 McDonald SJ, Middleton P, Dowswell T, et al. Effect of timing of umbilical cord clamping of term infants on maternal and neonatal outcomes. Cochrane Database Syst Rev 2013;7:CD004074.

7 Bhatt S, Polglase GR, Wallace EM, et al. Ventilation before umbilical cord clamping improves the physiological transition at birth. Front Pediatr 2014;2:113.

8 Gao Y, Raj JU. Regulation of the pulmonary circulation in the fetus and newborn. Physiol Rev 2010;90:1291-335

9 Polglase GR, Wallace MJ, Grant DA, et al. Influence of fetal breathing movements on pulmonary hemodynamics in fetal sheep. Pediatr Res 2004;56:932-8.

10 Sutton MS, Groves A, MacNeill A, et al. Assessment of changes in blood flow through the lungs and foramen ovale in the normal human fetus with gestational age: a prospective Doppler echocardiographic study. Br Heart J 1994;71:232-7.

11 Seed M, van Amerom JF, Yoo SJ, et al. Feasibility of quantification of the distribution of blood flow in the normal human fetal circulation using CMR a cross-sectional study. J Cardiovasc Magn Reson 2012;14:79.

12 Rudolph AM. Distribution and regulation of blood flow in the fetal and neonatal lamb. Circ Res 1985;57:811-21.

13 Hooper SB, Harding R. Fetal lung liquid: a major determinant of the growth and functional development of the fetal lung. Clin Exp Pharmacol Physiol 1995:22:235-47

14 Hooper SB, Siew ML, Kitchen MJ, et al. Establishing functional residual capacity in the non-breathing infant. Semin Fetal Neonatal Med 2013;18:336-43.

15 te Pas $A B$, Davis PG, Hooper $S B$, et al. From liquid to air: breathing after birth. J Pediatr 2008;152:607-11.

16 Hooper SB, Kitchen MJ, Wallace MJ, et al. Imaging lung aeration and lung liquid clearance at birth. FASEB J 2007;21:3329-37. 
17 Siew ML, Wallace MJ, Allison BJ, et al. The role of lung inflation and sodium transport in airway liquid clearance during lung aeration in newborn rabbits. Pediatr Res 2013;73(4 Pt 1):443-9.

18 Siew ML, Wallace MJ, Kitchen MJ, et al. Inspiration regulates the rate and temporal pattern of lung liquid clearance and lung aeration at birth. J Appl Physiol 2009;106:1888-95.

19 Bland RD, Bressack MA, McMillan DD. Labor decreases the lung water content of newborn rabbits. Am J Obstet Gynecol 1979;135:364-7.

20 Polglase GR, Morley CJ, Crossley KJ, et al. Positive end-expiratory pressure differentially alters pulmonary hemodynamics and oxygenation in ventilated, very premature lambs. J Appl Physiol 2005;99:1453-61.

21 Morin FC III, Egan EA, Ferguson W, et al. Development of pulmonary vascular response to oxygen. Am J Physiol 1988;254(3 Pt 2):H542-46.

22 Lang JA, Pearson JT, te Pas AB, et al. Ventilation/perfusion mismatch during lung aeration at birth. J Appl Physiol (1985) 2014;117:535-43.

23 Teitel DF, Iwamoto HS, Rudolph AM. Changes in the pulmonary circulation during birth-related events. Pediatr Res 1990;27(Pt 1):372-8.

24 Sobotka KS, Hooper SB, Allison BJ, et al. An initial sustained inflation improves the respiratory and cardiovascular transition at birth in preterm lambs. Pediatr Res 2011;70:56-60.

25 Dawes GS, Mott JC, Widdicombe JG. Closure of the foramen ovale in newborn lambs. J Physiol 1955;128:384-95.

26 Evans N, lyer P. Incompetence of the foramen ovale in preterm infants supported by mechanical ventilation. J Pediatr 1994;125(Pt 1):786-92.

27 van Vonderen JJ, Roest AA, Siew ML, et al. Noninvasive measurements of hemodynamic transition directly after birth. Pediatr Res 2014;75:448-52.

28 van Vonderen JJ, te Pas AB, Kolster-Bijdevaate $C$, et al. Non-invasive measurements of ductus arteriosus flow directly after birth. Arch Dis Child Fetal Neonatal Ed 2014;99:F408-12.
29 Reuss ML, Rudolph AM. Distribution and recirculation of umbilical and systemic venous blood flow in fetal lambs during hypoxia. I Dev Physiol 1980;2:71-84.

30 Bocking AD, Gagnon R, White $S E$, et al. Circulatory responses to prolonged hypoxemia in fetal sheep. Am J Obstet Gynecol 1988;159:1418-24.

31 Polglase GR, Miller SL, Barton SK, et al. Respiratory support for premature neonates in the delivery room: effects on cardiovascular function and the development of brain injury. Pediatr Res 2014;75:682-8.

32 Begley CM, Gyte GML, Devane D, et al. Active versus expectant management for women in the third stage of labour. Cochrane Database Syst Rev 2011(11): CD007412.

33 Soltani $H$, Hutchon DR, Poulose TA. Timing of prophylactic uterotonics for the third stage of labour after vaginal birth. Cochrane Database Syst Rev 2010;(8): CD006173.

34 Sheldon WR, Durocher J, Winikoff B, et al. How effective are the components of active management of the third stage of labor? BMC Pregnancy Childbirth 2013;13:46.

35 Yao $\mathrm{AC}$, Hirvensalo $\mathrm{M}$, Lind J. Placental transfusion-rate and uterine contraction. Lancet 1968;1:380-3.

36 Westgate JA, Wibbens $B$, Bennet $L$, et al. The intrapartum deceleration in center stage: a physiologic approach to the interpretation of fetal heart rate changes in labor. Am J Obstet Gynecol 2007;197:236.e1-11.

37 Yao AC, Lind J. Effect of gravity on placental transfusion. Lancet 1969;2:505-8.

38 Yao $A C$, Lind J. Blood flow in the umbilical vessels during the third stage of labor. Biol Neonate 1974;25:186-93.

39 Vain NE, Satragno DS, Gorenstein AN, et al. Effect of gravity on volume of placental transfusion: a multicentre, randomised, non-inferiority trial. Lancet 2014;384:235-40. 